\title{
Convergence of neutral type SICNNs involving proportional delays and $D$ operators
}

\author{
Renwei Jia ${ }^{1}$ and Shuhua Gong ${ }^{2^{*}}$ (D)
}

\section{"Correspondence:}

shuhuagong@aliyun.com

${ }^{2}$ College of Mathematics, Physics and Information Engineering, Jiaxing University, Jiaxing, P.R. China Full list of author information is available at the end of the article

\begin{abstract}
In the present study, by employing differential inequality theory, some novel assertions are gained to validate the global exponential convergence on neutral type shunting inhibitory cellular neural networks involving proportional delays and $D$ operators. Moreover, numerical simulations are provided to support the effectiveness of the analytical results.
\end{abstract}

MSC: 92C42; 93D20; 94D05; 65L20

Keywords: Exponential convergence; Neutral type shunting inhibitory cellular neural network; Proportional delay; D operator

\section{Introduction}

The well-known shunting inhibitory cellular neural networks (SICNNs), first proposed by Bouzerdoum and Pinter [1], have been extensively studied both in theory and applications [2-7]. In particular, qualitative and stability analysis for SICNNs with neutral type delays plays an important role in the design and applications of neural networks [8-11]. Usually, all neutral type SICNNs models can be converted into non-operator-based neutral functional differential equations (NFDEs) [8-10] and D-operator-based NFDEs [11], respectively.

In the past two decades, proportional delays occurring in nonlinear dynamics have attracted considerable attention because of their potential applications in various aspects such as web quality of service routing decision, collection of current of electric locomotive, nonlinear dynamical behavior, electrodynamics and principle of probability (see [1215]). However, so far, there is existing few articles on the global exponential convergence of neutral type SICNNs involving proportional delays and $D$ operators [16].

Inspired by the above viewpoint, in this article, our goal is to study the global exponential convergence for the following neutral type SICNNs involving proportional delays and $D$ operators:

$$
\begin{aligned}
& {\left[x_{i j}(t)-p_{i j}(t) x_{i j}\left(r_{i j} t\right)\right]^{\prime}} \\
& \quad=-a_{i j}(t) x_{i j}(t)-\sum_{C_{k l} \in N_{r}(i, j)} C_{i j}^{k l}(t) f\left(x_{k l}\left(q_{k l} t\right)\right) x_{i j}(t)+L_{i j}(t), \quad t \geq 1,
\end{aligned}
$$

(c) The Author(s) 2018. This article is distributed under the terms of the Creative Commons Attribution 4.0 International License (http://creativecommons.org/licenses/by/4.0/), which permits unrestricted use, distribution, and reproduction in any medium, provided you give appropriate credit to the original author(s) and the source, provide a link to the Creative Commons license, and indicate if changes were made. 
where $i j \in J=\{11,12, \ldots, 1 n, \ldots, m 1, m 2, \ldots, m n\}, m n$ corresponds to the number of units in a neural network, $C_{i j}$ is the cell at the position $(i, j)$ of the lattice, $N_{r}(i, j)=\left\{C_{k l}: \max (\mid k-\right.$ $i|| l-j \mid,) \leq r, 1 \leq k \leq m, 1 \leq l \leq n\}$ is the $r$ neighborhood of $C_{i j}$, proportional delay factors $q_{k l}$ and $r_{i j}$ satisfy the conditions that $0<q_{k l}, r_{i j}<1$. Further information on the activation functions and coefficient parameters is available from $[9,11]$.

Throughout the rest of this article, the following concepts and notations will be adopted. For any $x=\left\{x_{i j}\right\}=\left(x_{i j}\right)_{1 \times m n} \in \mathbb{R}^{m n}$,

$$
\begin{aligned}
& |x|=\left\{\left|x_{i j}\right|\right\}, \quad\|x(t)\|=\max _{i j \in J}\left|x_{i j}(t)\right|, \\
& \rho_{i j}=\min \left\{r_{i j}, q_{i j}\right\}, \quad r=\frac{1}{\max _{i j \in J} \max \left\{q_{i j}, r_{i j}\right\}}, \\
& W^{+}=\sup _{t \in \mathbb{R}}|W(t)|, \quad W^{-}=\inf _{t \in \mathbb{R}}|W(t)| .
\end{aligned}
$$

The initial condition involved in systems (1.1) can be described as follows:

$$
x_{i j}(s)=\varphi_{i j}(s), \quad s \in\left[\rho_{i j}, 1\right], \varphi_{i j} \in C\left(\left[\rho_{i j}, 1\right], \mathbb{R}\right), i j \in J .
$$

Furthermore, it is assumed that $a_{i j}, p_{i j}, L_{i j}, C_{i j}^{k l} \in B C\left(\left[\rho_{i j},+\infty\right), \mathbb{R}\right)$, where $B C\left(\left[\rho_{i j},+\infty\right), \mathbb{R}\right)$ designates the set of bounded and continuous functions, and $i j \in J$.

In addition, for $i j \in J$, the following hypotheses will be imposed:

$\left(\mathrm{S}_{0}\right)$ There exist $\tilde{a}_{i j} \in B C(\mathbb{R},(0,+\infty))$ and $K_{i j}>0$ satisfying

$$
e^{-\int_{s}^{t} a_{i j}(u) d u} \leq K_{i j} e^{-\int_{s}^{t} \tilde{a}_{i j}(u) d u} \quad \forall t, s \in \mathbb{R}, t-s \geq 0 .
$$

$\left(\mathrm{S}_{1}\right) f \in C[\mathbb{R}, \mathbb{R}], \sup _{u \in \mathbb{R}}|f(u)|=M^{f} \geq 0$.

$\left(\mathrm{S}_{2}\right)$ There are constants $H_{i j}, \lambda_{0} \in(0,+\infty)$ obeying

$$
H_{i j}=\sup _{s \geq \rho_{i j}}\left|p_{i j}(s)\right| e^{\lambda_{0}\left(1-r_{i j}\right) s}<1, \quad L_{i j}(t)=O\left(e^{-\lambda_{0} t}\right) \quad \text { as } t \rightarrow+\infty,
$$

and

$$
\sup _{t \geq 1}\left\{-\tilde{a}_{i j}(t)+K_{i j}\left[\frac{e^{\lambda_{0}\left(1-r_{i j}\right) t}}{1-H_{i j}}\left|a_{i j}(t) p_{i j}(t)\right|+\sum_{C_{k l} \in N_{r}(i, j)}\left|C_{i j}^{k l}(t)\right| M^{f} \frac{1}{1-H_{i j}}\right]\right\}<0 .
$$

\section{Global existence and convergence of solutions}

In this section, we will validate the global existence and convergence of every solution for SICNNs (1.1) with initial condition (1.2).

Lemma 2.1 If $\left(\mathrm{S}_{0}\right),\left(\mathrm{S}_{1}\right)$ and $\left(\mathrm{S}_{2}\right)$ are obeyed, then every solution $x(t)$ of $(1.1)-(1.2)$ exists and is unique on $[1,+\infty)$.

Proof For $i j \in J$ and $t \in[1, r]$, let

$$
y_{i j}(t)=x_{i j}(t)-p_{i j}(t) x_{i j}\left(r_{i j} t\right), \quad \beta_{i j}(t)=p_{i j}(t) \varphi_{i j}\left(r_{i j} t\right)
$$


and

$$
A_{i j}(t)=a_{i j}(t)+\sum_{C_{k l} \in N_{r}(i, j)} C_{i j}^{k l}(t) f\left(\varphi_{k l}\left(q_{k l} t\right)\right), \quad B_{i j}(t)=-A_{i j}(t) p_{i j}(t) \varphi_{i j}\left(r_{i j} t\right)+L_{i j}(t)
$$

Then

$$
\begin{aligned}
y_{i j}^{\prime}(t) & =\left[x_{i j}(t)-p_{i j}(t) x_{i j}\left(r_{i j} t\right)\right]^{\prime} \\
& =-a_{i j}(t) x_{i j}(t)-\sum_{C_{k l} \in N_{r}(i, j)} C_{i j}^{k l}(t) f\left(x_{k l}\left(q_{k l} t\right)\right) x_{i j}(t)+L_{i j}(t) \\
& =-\left[a_{i j}(t)+\sum_{C_{k l} \in N_{r}(i, j)} C_{i j}^{k l}(t) f\left(\varphi_{k l}\left(q_{k l} t\right)\right)\right] x_{i j}(t)+L_{i j}(t) \\
& =-A_{i j}(t) y_{i j}(t)+B_{i j}(t), \quad t \in[1, r] .
\end{aligned}
$$

From (2.1), by using a similar argument as in the proof Lemma 2.2 in [16], one can prove that $x(t)=y(t)+\left\{\beta_{i j}(t)\right\}$ exists and is unique on $[1, r],\left[r, r^{2}\right],\left[r^{2}, r^{3}\right], \ldots$. This finishes the proofs of Lemma 2.1. This finishes the proof of Lemma 2.1.

Theorem 2.1 Assume that all hypotheses mentioned in Sect. 1 hold. Then, there is a constant $\lambda \in\left(0, \lambda_{0}\right)$ such that

$$
x_{i j}(t)=O\left(e^{-\lambda t}\right) \quad \text { as } t \rightarrow+\infty, i j \in J
$$

where $x(t)=\left\{x_{i j}(t)\right\}$ is an arbitrary solution vector of the initial value problem (1.1)-(1.2).

Proof We trivially extend $x(t)$ to $\left[r_{i j} \rho_{i j},+\infty\right)$ by setting $x_{i j}(t)=\varphi_{i j}(t)=\varphi_{i j}\left(\rho_{i j}\right)$ for $t \in$ $\left[r_{i j} \rho_{i j}, \rho_{i j}\right], i j \in J$. Let

$$
X_{i j}(t)=x_{i j}(t)-p_{i j}(t) x_{i j}\left(r_{i j} t\right), \quad \text { for all } t \in\left[\rho_{i j},+\infty\right), i j \in J .
$$

Then, $x_{i j}(t)$ and $X_{i j}(t)$ are continuous on $\left[\rho_{i j}, 1\right]$, and

$$
\begin{aligned}
X_{i j}^{\prime}(t)= & {\left[x_{i j}(t)-p_{i j}(t) x_{i j}\left(r_{i j} t\right)\right]^{\prime} } \\
= & -a_{i j}(t) X_{i j}(t)-a_{i j}(t) p_{i j}(t) x_{i j}\left(r_{i j} t\right) \\
& -\sum_{C_{k l} \in N_{r}(i, j)} C_{i j}^{k l}(t) f\left(x_{k l}\left(q_{k l} t\right)\right) x_{i j}(t)+L_{i j}(t), \quad t \geq 1, i j \in J .
\end{aligned}
$$

In view of $\left(\mathrm{S}_{2}\right)$, we can take $\lambda \in\left(0, \min \left\{\lambda_{0}, \min _{i j \in J} \tilde{a}_{i j}^{-}\right\}\right)$obeying

$$
\begin{aligned}
& \sup _{t \geq 1}\left\{\lambda-\tilde{a}_{i j}(t)+K_{i j}\left[\frac{e^{\lambda\left(1-r_{i j}\right) t}}{1-H_{i j}}\left|a_{i j}(t) p_{i j}(t)\right|+\sum_{C_{k l} \in N_{r}(i, j)}\left|C_{i j}^{k l}(t)\right| M^{f} \frac{1}{1-H_{i j}}+\lambda\right]\right\} \\
& \leq \sup _{t \geq 1}\left\{\lambda-\tilde{a}_{i j}(t)+K_{i j}\left[\frac{e^{\lambda_{0}\left(1-r_{i j}\right) t}}{1-H_{i j}}\left|a_{i j}(t) p_{i j}(t)\right|\right.\right. \\
& \left.\left.\quad+\sum_{C_{k l} \in N_{r}(i, j)}\left|C_{i j}^{k l}(t)\right| M^{f} \frac{1}{1-H_{i j}}+\lambda\right]\right\}<0, \quad i j \in J .
\end{aligned}
$$


With no loss of generality, let

$$
\|\varphi\|_{X}=\max _{i j \in J} \sup _{t \in\left[\rho_{i j}, 1\right]}\left|\varphi_{i j}(t)-p_{i j}(t) \varphi_{i j}\left(r_{i j} t\right)\right|>0 .
$$

For any $\varepsilon>0$, we can pick an $\varepsilon$-independent constant $M$ such that

$$
M=1+\max _{i j \in J} K_{i j}, \quad\left|L_{i j}(t)\right|<\lambda M\left(\|\varphi\|_{X}+\varepsilon\right) e^{-\lambda(t-1)} \quad \text { for all } t \in[1,+\infty), i j \in J
$$

which leads to

$$
\|X(1)\|<\left(\|\varphi\|_{X}+\varepsilon\right)
$$

and

$$
\left|X_{i j}(t)\right|<\left(\|\varphi\|_{X}+\varepsilon\right) e^{-\lambda(t-1)}<M\left(\|\varphi\|_{X}+\varepsilon\right) e^{-\lambda(t-1)} \quad \text { for all } t \in\left[\rho_{i j}, 1\right], i j \in J .
$$

Hereafter, we will validate

$$
\|X(t)\|<M\left(\|\varphi\|_{X}+\varepsilon\right) e^{-\lambda(t-1)} \quad \text { for all } t>1 .
$$

In the contrary case, there must exist $i j \in J$ and $\theta>1$ obeying

$$
\|X(\theta)\|=\left|X_{i j}(\theta)\right|=M\left(\|\varphi\|_{X}+\varepsilon\right) e^{-\lambda(\theta-1)}
$$

and

$$
\left|X_{k l}(t)\right|<M\left(\|\varphi\|_{X}+\varepsilon\right) e^{-\lambda(t-1)} \quad \text { for all } t \in\left[\rho_{k l}, \theta\right), k l \in J
$$

From the fact that

$$
\begin{aligned}
e^{\lambda v}\left|x_{k l}(v)\right| & \leq e^{\lambda v}\left|x_{k l}(v)-p_{k l}(v) x_{k l}\left(r_{k l} v\right)\right|+e^{\lambda v}\left|p_{k l}(v) x_{k l}\left(r_{k l} v\right)\right| \\
& \leq e^{\lambda v}\left|X_{k l}(v)\right|+\left|p_{k l}(v)\right| e^{\lambda\left(1-r_{k l}\right) v} e^{\lambda r_{k l} v}\left|x_{k l}\left(r_{k l} v\right)\right| \\
& \leq e^{\lambda v}\left|X_{k l}(v)\right|+\sup _{s \geq \rho_{k l}}\left|p_{k l}(s)\right| e^{\lambda\left(1-r_{k l}\right) s} \sup _{s \in\left[r_{k l} \rho_{k l}, r_{k l} t\right]} e^{\lambda s}\left|x_{k l}(s)\right| \\
& \leq e^{\lambda v}\left|X_{k l}(v)\right|+\sup _{s \geq \rho_{k l}}\left|p_{k l}(s)\right| e^{\lambda\left(1-r_{k l}\right) s} \sup _{s \in\left[\rho_{k l}, t\right]} e^{\lambda s}\left|x_{k l}(s)\right|,
\end{aligned}
$$

we obtain

$$
\begin{aligned}
e^{\lambda t}\left|x_{k l}(t)\right| & \leq \sup _{s \in\left[\rho_{k l}, t\right]} e^{\lambda s}\left|x_{k l}(s)\right| \leq \frac{M\left(\|\varphi\|_{X}+\varepsilon\right) e^{\lambda}}{1-\sup _{s \geq \rho_{k l}}\left|p_{k l}(s)\right| e^{\lambda_{0}\left(1-r_{k l}\right) s}} \\
& =\frac{M\left(\|\varphi\|_{X}+\varepsilon\right) e^{\lambda}}{1-H_{k l}}
\end{aligned}
$$


where $v \in\left[\rho_{k l}, t\right], t \in[1, \theta), k l \in J$. Together with (2.4), (2.5), (2.6), (2.9) and (2.11), we conclude that

$$
\begin{aligned}
& \left|X_{i j}(\theta)\right|=\mid X_{i j}(1) e^{-\int_{1}^{\theta} a_{i j}(u) d u}+\int_{1}^{\theta} e^{-\int_{t}^{\theta} a_{i j}(u) d u}\left[-a_{i j}(t) p_{i j}(t) x_{i j}\left(r_{i j} t\right)\right. \\
& \left.-\sum_{C_{k l} \in N_{r}(i, j)} C_{i j}^{k l}(t) f\left(x_{k l}\left(q_{k l} t\right)\right) x_{i j}(t)+L_{i j}(t)\right] d t \\
& \leq\left|X_{i j}(1)\right| K_{i j} e^{-\int_{1}^{\theta} \tilde{a}_{i j}(u) d u}+\int_{1}^{\theta} e^{-\int_{t}^{\theta} \tilde{a}_{i j}(u) d u} K_{i j} \mid-a_{i j}(t) p_{i j}(t) x_{i j}\left(r_{i j} t\right) \\
& -\sum_{C_{k l} \in N_{r}(i, j)} C_{i j}^{k l}(t) f\left(x_{k l}\left(q_{k l} t\right)\right) x_{i j}(t)+L_{i j}(t) \mid d t \\
& \leq\left(\|\varphi\|_{X}+\varepsilon\right) K_{i j} e^{-\int_{1}^{\theta} \tilde{a}_{i j}(u) d u}+\int_{1}^{\theta} e^{-\int_{t}^{\theta} \tilde{a}_{i j}(u) d u} K_{i j}\left[\left|a_{i j}(t) p_{i j}(t)\right|\left|x_{i j}\left(r_{i j} t\right)\right|\right. \\
& \left.+\sum_{C_{k l} \in N_{r}(i, j)}\left|C_{i j}^{k l}(t)\right| M^{f}\left|x_{i j}(t)\right|+\left|L_{i j}(t)\right|\right] d s \\
& \leq\left(\|\varphi\|_{X}+\varepsilon\right) e^{-\lambda(\theta-1)} K_{i j} e^{-\int_{1}^{\theta}\left[\tilde{a}_{i j}(u)-\lambda\right] d u} \\
& +\int_{1}^{\theta} e^{-\int_{t}^{\theta}\left[\tilde{a}_{i j}(u)-\lambda\right] d u} K_{i j}\left[\frac{e^{\lambda\left(1-r_{i j}\right) t}}{1-H_{i j}}\left|a_{i j}(t) p_{i j}(t)\right|\right. \\
& \left.+\sum_{C_{k l} \in N_{r}(i, j)}\left|C_{i j}^{k l}(t)\right| M^{f} \frac{1}{1-H_{i j}}+\lambda\right] d t M\left(\|\varphi\|_{X}+\varepsilon\right) e^{-\lambda(\theta-1)} \\
& \leq\left(\|\varphi\|_{X}+\varepsilon\right) e^{-\lambda(\theta-1)} K_{i j} e^{-\int_{1}^{\theta}\left[\tilde{a}_{i j}(u)-\lambda\right] d u}
\end{aligned}
$$

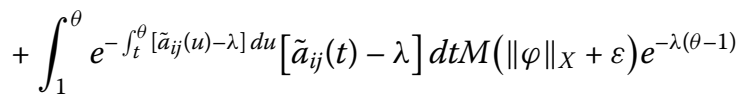

$$
\begin{aligned}
& =M\left(\|\varphi\|_{X}+\varepsilon\right) e^{-\lambda(\theta-1)}\left[\left(\frac{K_{i j}}{M}-1\right) e^{-\int_{1}^{\theta}\left(\tilde{a}_{i j}(u)-\lambda\right) d u}+1\right] \\
& <M\left(\|\varphi\|_{X}+\varepsilon\right) e^{-\lambda(\theta-1)} \text {. }
\end{aligned}
$$

This is a clear contradiction of (2.8). Thus, (2.7) is true. Letting $\varepsilon \rightarrow 0^{+}$suggests

$$
\|X(t)\| \leq M\|\varphi\|_{X} e^{-\lambda(t-1)} \quad \text { for all } t>1 .
$$

Then, using a similar theoretical derivation as in the proof of (2.10) and (2.11), gives us

$$
e^{\lambda t}\left|x_{i j}(t)\right| \leq \sup _{s \in\left[\rho_{i j}, t\right]} e^{\lambda s}\left|x_{i j}(s)\right| \leq \frac{M\|\varphi\|_{X} e^{\lambda}}{1-H_{i j}}
$$

and

$$
\left|x_{i j}(t)\right| \leq \frac{M\|\varphi\|_{X}}{1-H_{i j}} e^{-\lambda(t-1)}, \quad \forall t>1, i j \in J .
$$

This completes the proof. 


\section{Simulation examples}

Example 3.1 Consider the following neutral type SICNNs:

$$
\begin{aligned}
& {\left[x_{i j}(t)-p_{i j}(t) x_{i j}\left(r_{i j} t\right)\right]^{\prime}} \\
& \quad=-a_{i j}(t) x_{i j}(t)-\sum_{C_{k l} \in N_{1}(i, j)} C_{i j}^{k l}(t) \frac{1}{10} \arctan \left(x_{k l}\left(q_{i j} t\right)\right) x_{i j}(t)+L_{i j}(t),
\end{aligned}
$$

where $p_{i j}(t)=\frac{1}{5} e^{-t} \sin (i+j) t, r_{i j}=q_{i j}=\frac{1}{2}, i, j=1,2$,

$$
\begin{aligned}
& {\left[\begin{array}{ll}
a_{11} & a_{12} \\
a_{21} & a_{22}
\end{array}\right]=\left[\begin{array}{cc}
0.8+\cos 100 t & 1+1.1 \sin 100 t \\
0.8+1.3 \cos 100 t & 1+1.2 \sin 100 t
\end{array}\right],} \\
& {\left[\begin{array}{ll}
C_{11} & C_{12} \\
C_{21} & C_{22}
\end{array}\right]=\left[\begin{array}{ll}
0.01 \cos 2 t & 0.02 \cos 3 t \\
0.02 \cos 3 t & 0.01 \cos 4 t
\end{array}\right],} \\
& {\left[\begin{array}{ll}
L_{11} & L_{12} \\
L_{21} & L_{22}
\end{array}\right]=\left\{\frac{i+j}{100} e^{-2|t|} \sin t\right\} .}
\end{aligned}
$$

Pick

$$
\begin{aligned}
& {\left[\begin{array}{ll}
\tilde{a}_{11} & \tilde{a}_{12} \\
\tilde{a}_{21} & \tilde{a}_{22}
\end{array}\right]=\left[\begin{array}{ll}
0.8 & 1 \\
0.8 & 1
\end{array}\right], \quad K_{i j} \leq e^{\frac{1}{25},} \quad M^{f}=\frac{\pi}{20},} \\
& \sum_{C_{k l} \in N_{1}(i, j)}\left|C_{i j}^{k l}(t)\right| \leq 0.06, \quad \lambda_{0}=1.8, \quad i, j=1,2,
\end{aligned}
$$

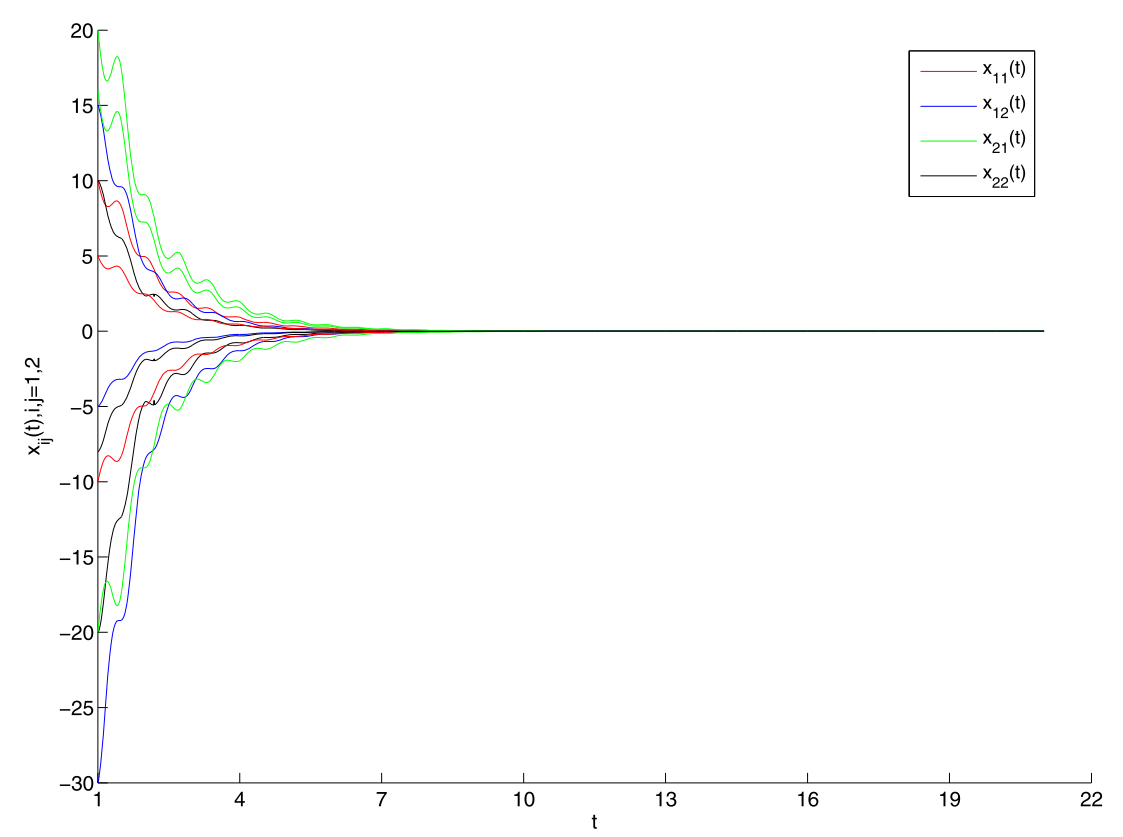

Figure 1 Numerical solutions of system (3.1) with different initial values 
such that

$$
\begin{aligned}
& \sup _{t \geq 1}\left\{-\tilde{a}_{i j}(t)+K_{i j}\left[\frac{e^{\lambda_{0}\left(1-r_{i j}\right) t}}{1-H_{i j}}\left|a_{i j}(t) p_{i j}(t)\right|+\sum_{C_{k l} \in N_{r}(i, j)}\left|C_{i j}^{k l}(t)\right| M^{f} \frac{1}{1-H_{i j}}\right]\right\} \\
& \quad<-0.2, \quad i, j=1,2 .
\end{aligned}
$$

Then, it is easy to verify that (3.1) obeys $\left(\mathrm{S}_{0}\right),\left(\mathrm{S}_{1}\right)$ and $\left(\mathrm{S}_{2}\right)$. Hence, by Theorem 2.1, we get that all solutions of system (3.1) converge exponentially to the zero vector with the exponential convergence rate $\lambda \approx 0.02$. Furthermore, we have the following simulation results shown in Fig. 1.

Remark 3.1 To the best of our knowledge, this is the first time when attention is focused on the global exponential convergence for neutral type SICNNs involving proportional delays and $D$ operators. Based on differential inequality theory, we show that all solutions of the addressed model are exponentially convergent to the zero vector under suitable hypotheses. In particular, we provide an upper bound for the exponential convergence rate. Most recently, the generalized exponential stability and pseudo almost periodicity of neutral type SICNNs have been established in $[16,17]$, and some other dynamical behaviors of neural networks have obtained in [18-20]. Unfortunately, the global exponential convergence for every solution of neutral type SICNNs involving proportional delays and $D$ operators has not been investigated in [16-20]. This suggests that all results in the references [16-20] cannot be straightly applied to show the exponential convergence on every solution in system (3.1).

\section{Acknowledgements}

This work was supported by the Natural Scientific Research Fund of Hunan Provincial Education Department of China (Grant No. 17C1076), the Zhejiang Provincial Natural Science Foundation of China (Grant No. LY18A010019) and the Zhejiang Provincial Education Department Natural Science Foundation of China (Y201533862).

\section{Funding}

This work was supported by the Natural Scientific Research Fund of Hunan Provincial Education Department of China (Grant No. 17C1076), the Zhejiang Provincial Natural Science Foundation of China (Grant No. LY18A010019) and the

Zhejiang Provincial Education Department Natural Science Foundation of China (Y201533862).

\section{Competing interests}

The authors declare that they have no competing interests.

Authors' contributions

RWJ and SHG worked together in the derivation of the mathematical results. Both authors read and approved the final manuscript.

\section{Author details}

${ }^{1}$ College of Mathematics and Computer Science, Hunan University of Arts and Science, Changde, P.R. China. ${ }^{2}$ College of Mathematics, Physics and Information Engineering, Jiaxing University, Jiaxing, P.R. China.

\section{Publisher's Note}

Springer Nature remains neutral with regard to jurisdictional claims in published maps and institutional affiliations.

Received: 27 June 2018 Accepted: 2 October 2018 Published online: 10 October 2018

\section{References}

1. Bouzerdoum, A., Pinter, R.B.: Analysis and analog implementation of directionally sensitive shunting inhibitory cellular neural networks. In: Visual Information Processing: From Neurons to Chips, vol. SPIE-1473, pp. $29-38$ (1991)

2. Bouzerdoum, A., Pinter, R.B.: Shunting inhibitory cellular neural networks: derivation and stability analysis. IEEE Trans. Circuits Syst. I, Fundam. Theory Appl. 40, 215-221 (1993)

3. Bouzerdoum, A., Pinter, R.B.: Nonlinear lateral inhibition applied to motion detection in the fly visual system. In: Pinter, R.B., Nabet, B. (eds.) Nonlinear Vision, pp. 423-450. CRC Press, Boca Raton (1992) 
4. Chen, Z:: A shunting inhibitory cellular neural network with leakage delays and continuously distributed delays of neutral type. Neural Comput. Appl. 23, 2429-2434 (2013)

5. Liu, X:: Exponential convergence of SICNNs with delays and oscillating coefficients in leakage terms. Neurocomputing 168, 500-504 (2015)

6. Jiang, A.: Exponential convergence for shunting inhibitory cellular neural networks with oscillating coefficients in leakage terms. Neurocomputing 165, 159-162 (2015)

7. Long, Z: New results on anti-periodic solutions for SICNNs with oscillating coefficients in leakage terms. Neurocomputing 171(1), 503-509 (2016)

8. Zhao, C., Wang, Z: Exponential convergence of a SICNN with leakage delays and continuously distributed delays of neutral type. Neural Process. Lett. 41, 239-247 (2015)

9. Liu, B.: Global exponential convergence of non-autonomous SICNNs with multi-proportional delays. Neural Comput. Appl. 28, 1927-1931 (2017)

10. Yu, Y.: Global exponential convergence for a class of neutral functional differential equations with proportional delays. Math. Methods Appl. Sci. 39, 4520-4525 (2016)

11. Yao, L.: Global exponential convergence of neutral type shunting inhibitory cellular neural networks with $D$ operator. Neural Process. Lett. 45, 401-409 (2017)

12. Ockendon, J.R., Tayler, A.B.: The dynamics of a current collection system for an electric locomotive. Proc. R. Soc. Lond., Ser. A, Math. Phys. Eng. Sci. 322(1551), 447-468 (1971)

13. Liu, B:: Global exponential convergence of non-autonomous cellular neural networks with multi-proportional delays. Neurocomputing 191, 352-355 (2016)

14. Liu, B.: Finite-time stability of CNNs with neutral proportional delays and time-varying leakage delays. Math. Methods Appl. Sci. 40, 167-174 (2017)

15. Yu, Y.: Global exponential convergence for a class of HCNNs with neutral time-proportional delays. Appl. Math. Comput. 285, 1-7 (2016)

16. Zhang, A.: Almost periodic solutions for SICNNs with neutral type proportional delays and D operators. Neural Process. Lett. 47, 57-70 (2018)

17. Zhang, A.: Pseudo almost periodic solutions for neutral type SICNNs with D operator. J. Exp. Theor. Artif. Intell. 29(4), 795-807 (2017)

18. Huang, C., Yang, Z., Yi, T., Zou, X.: On the basins of attraction for a class of delay differential equations with non-monotone bistable nonlinearities. J. Differ. Equ. 256(7), 2101-2114 (2014)

19. Huang, C., Cao, J., Cao, J.D.: Stability analysis of switched cellular neural networks: A mode-dependent average dwell time approach. Neural Netw. 82, 84-99 (2016)

20. Huang, C., Liu, B., Tian, X., Yang, L.: Global convergence on asymptotically almost periodic SICNNs with nonlinear decay functions. Neural Process. Lett. (2018). https://doi.org/10.1007/s11063-018-9835-3

\section{Submit your manuscript to a SpringerOpen ${ }^{\circ}$ journal and benefit from:}

- Convenient online submission

- Rigorous peer review

- Open access: articles freely available online

- High visibility within the field

- Retaining the copyright to your article

Submit your next manuscript at $\boldsymbol{\nabla}$ springeropen.com 